\title{
Electronic structure of monolayer antimonene nanoribbons under out-of-plane and transverse bias
}

\author{
Edo van Veen, ${ }^{1}$ Jin Yu, ${ }^{2,1}$ Mikhail I. Katsnelson, ${ }^{1}$ Rafael Roldán, ${ }^{3}$ and Shengjun Yuan ${ }^{4, *}$ \\ ${ }^{1}$ Institute for Molecules and Materials, Radboud University, NL-6525 AJ Nijmegen, The Netherlands \\ ${ }^{2}$ Beijing Computational Science Research Center, Beijing 100094, China \\ ${ }^{3}$ Materials Science Factory, Instituto de Ciencia de Materiales de Madrid (ICMM), \\ Consejo Superior de Investigaciones Científicas (CSIC), Cantoblanco E28049 Madrid, Spain \\ ${ }^{4}$ School of Physics and Technology, Wuhan University, Wuhan 430072, China
}

(Received 24 June 2018; revised manuscript received 5 October 2018; published 29 November 2018)

\begin{abstract}
A systematic study of the electronic properties of single-layer $\mathrm{Sb}$ (antimonene) nanoribbons is presented. By using a six-orbital tight-binding Hamiltonian, we study the electronic band structure of finite ribbons with zigzag or armchair termination. We show that there is good agreement between ab initio calculations and the tight-binding model. We study how the size of the gap can be controlled by applying an external bias potential. An electric field applied perpendicular to the antimonene layer is found to increase the band gap, while a transverse bias potential leads to a position-dependent reduction of the band gap. Both kinds of bias potential break inversion symmetry of the crystal. This, together with the strong intrinsic spin-orbit coupling of antimonene, leads to spin splitting of the valence-band states.
\end{abstract}

DOI: 10.1103/PhysRevMaterials.2.114011

\section{INTRODUCTION}

Two-dimensional (2D) materials [1], such as graphene, transition-metal dichalcogenides, and hexagonal boron nitride, are attracting tremendous interest due to their unique electronic, optical, and mechanical properties, remarkably different from their three-dimensional counterparts [2]. Recently, the family of 2D materials derived from the group-VA layered crystals (P, As, Sb, Bi) has been the focus of great attention [3,4], with black phosphorus being the most well studied among them. In 2015, Zhang et al. predicted that contrary to bulk antimony which is a semimetal, single-layer $\mathrm{Sb}$ (antimonene) is an indirect band-gap semiconductor [5]. Soon after, it was demonstrated that atomically thin antimonene can be obtained by different means, including van der Waals epitaxy [6], micromechanical exfoliation [7], liquid phase exfoliation [8], molecular-beam epitaxy [9], or electrochemical exfoliation [10]. Theoretical calculations have studied in detail the electronic properties of this material [11-15]. Strong spin-orbit coupling was also reported, with a coupling strength of $\lambda \approx 0.34 \mathrm{eV}$ [16]. Ab initio quantum transport calculations have shown that antimonene field-effect transistors (FETs) can satisfy both the low-power and high-performance requirements for usage in nanoscale electronic and optoelectronic devices [17]. Previous experience with graphene and other 2D materials has further motivated theoretical studies of the electronic properties of nanoribbons of group-VA semiconductors [18-22]. Recently, experimental fabrication of antimonene nanoribbons has been reported [23], demonstrating band-gap opening due to quantum confinement.

In this work, we study the band structure and electronic properties of $\mathrm{Sb}$ nanoribbons in the presence of out-of-plane and in-plane electric fields. We find that edge states are present

\footnotetext{
*s.yuan@whu.edu.cn
}

in nanoribbons with both zigzag and armchair termination. We find good agreement between $a b$ initio numerical simulations and tight-binding calculations. We further demonstrate that the size of the band gap can be controlled by the presence of an external bias field. Application of a bias field breaks inversion symmetry which, together with the strong spin-orbit coupling in antimonene, leads to splitting of the valence-band edges, with the corresponding spin-valley coupling due to the Rashba effect.

The paper is organized as follows. In Sec. II, we describe the tight-binding model and the details of the calculations. We also show results for unbiased nanoribbons. In Sec. III, we study the effect of a perpendicular electric field on the electronic properties and the band structure of $\mathrm{Sb}$ nanoribbons, and the effect of an in-plane bias field is studied in Sec. IV. Our main results are summarized in Sec. V.

\section{MODEL AND METHOD}

Single-layer antimonene consists of a buckled honeycomb lattice of $\mathrm{Sb}$ atoms (Fig. 1), with the two sublattices vertically displaced by $b=1.65 \AA$, and with an in-plane lattice constant of $a=4.12 \AA$. The relevant energy bands of the electronic structure, including spin-orbit coupling (SOC) effects, are very well captured by a six-orbital tight-binding Hamiltonian developed by Rudenko et al. [16], which includes the three $p$ orbitals of each of the two $\mathrm{Sb}$ atoms of the unit cell,

$$
\begin{aligned}
H= & \sum_{m} \sum_{i} \sum_{\sigma} \epsilon_{m i \sigma} c_{m i \sigma}^{\dagger} c_{m i \sigma} \\
& +\sum_{m n} \sum_{i j} \sum_{\sigma} t_{m i \sigma ; n j \sigma} c_{m i \sigma}^{\dagger} c_{n j \sigma} \\
& +\sum_{m n} \sum_{i} \sum_{\sigma \sigma^{\prime}} h_{m i \sigma ; n i \sigma^{\prime}} c_{m i \sigma}^{\dagger} c_{n i \sigma^{\prime}},
\end{aligned}
$$



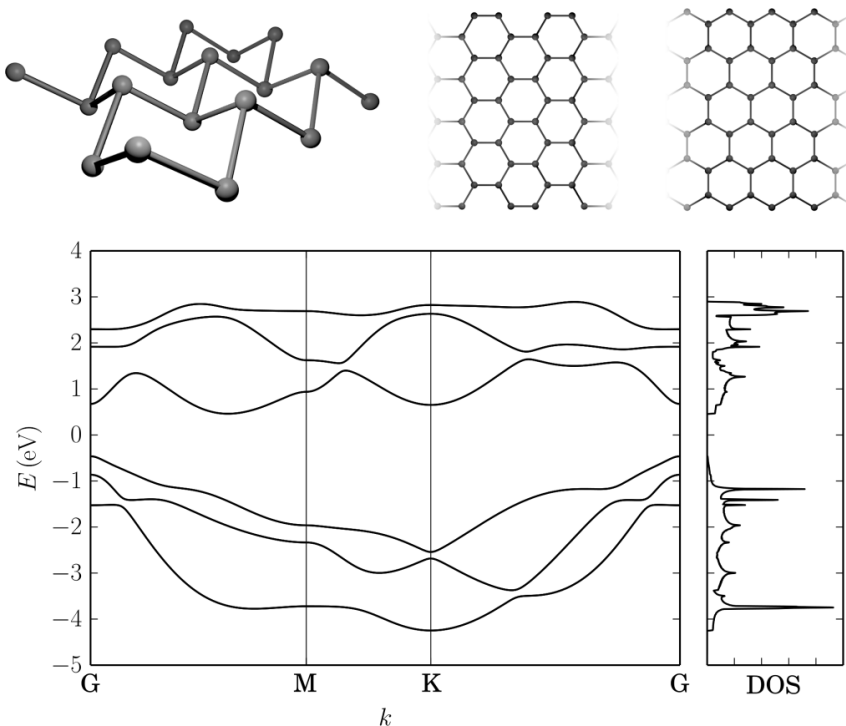

FIG. 1. Top: The buckled honeycomb lattice structure of antimony (left), an armchair SbNR (middle), and a zigzag SbNR (right). Bottom: Band structure and DOS for pristine Sb calculated with the tight-binding Hamiltonian (1), with the hopping parameters given in Table I.

where $m, n$ run over orbitals, $i, j$ run over sites, and $\sigma, \sigma^{\prime}$ run over spins; $c_{m i \sigma}^{\dagger}\left(c_{m i \sigma}\right)$ is the creation (annihilation) operator on orbital $m$ at site $i$ with spin $\sigma$. The parameters $\epsilon_{m i \sigma}$ account for on-site potentials, $t_{m i \sigma ; n j \sigma}$ are interorbital hopping terms, and intra-atomic SOC is accounted by $h_{m i \sigma ; n i \sigma^{\prime}}$. The intra-atomic SOC constant is $\lambda=0.34 \mathrm{eV}$ and the hopping parameters are given in Table I [16] and schematically shown in Fig. 2. The corresponding density of states (DOS) is calculated from

$$
D(E)=\frac{1}{2 \pi} \sum_{n} \int_{B Z} \delta\left[E-E_{n}(k)\right] d k,
$$

where $n$ labels the different energy bands. The band structure and DOS obtained with this model for bulk antimonene are shown in Fig. 1. Single-layer antimonene is an indirect gap semiconductor with a band gap of $0.92 \mathrm{eV}$. The edge of the valence band is located at the $\Gamma$ point of the Brillouin zone (BZ), with main contributions from $p_{x}$ and $p_{y}$ orbitals, while the edge of the conduction band is placed at a non-high-

TABLE I. Hopping amplitudes $t_{i}$ (in $\mathrm{eV}$ ) entering in the TB Hamiltonian given by Eq. (1), as obtained in Ref. [16]. $d$ denotes the distance between the lattice sites on which the interacting orbitals are centered. $N_{c}$ is the corresponding coordination number. The hoppings are schematically shown in Fig. 2.

\begin{tabular}{lrcccccccccc}
\hline \hline$i$ & $t_{i}(\mathrm{eV})$ & $d(\AA)$ & $N_{c}$ & $i$ & $t_{i}(\mathrm{eV})$ & $d(\AA)$ & $N_{c}$ & $i$ & $t_{i}(\mathrm{eV})$ & $d(\AA)$ & $N_{c}$ \\
\hline 1 & -2.09 & 2.89 & 1 & 6 & 0.21 & 4.12 & 1 & 11 & -0.06 & 4.12 & 2 \\
2 & 0.47 & 2.89 & 2 & 7 & 0.08 & 2.89 & 2 & 12 & -0.06 & 5.03 & 1 \\
3 & 0.18 & 4.12 & 4 & 8 & -0.07 & 5.03 & 2 & 13 & -0.03 & 6.50 & 2 \\
4 & -0.50 & 4.12 & 1 & 9 & 0.07 & 6.50 & 2 & 14 & -0.04 & 8.24 & 1 \\
5 & -0.11 & 6.50 & 2 & 10 & 0.07 & 6.50 & 2 & 15 & -0.03 & 8.24 & 1 \\
\hline \hline
\end{tabular}

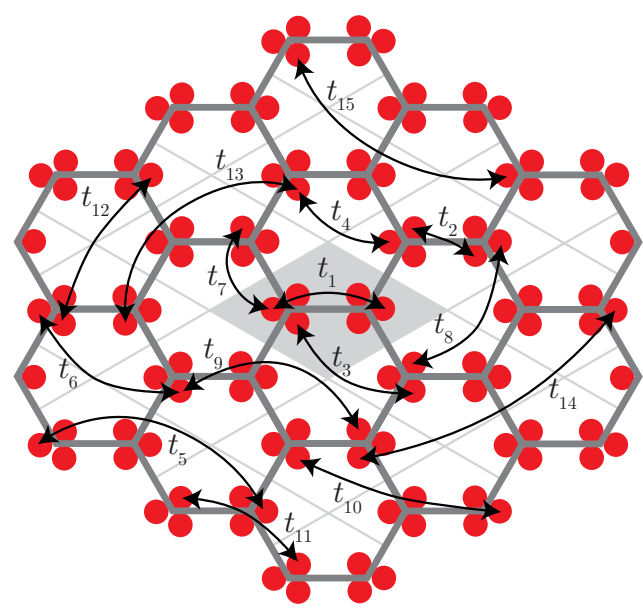

FIG. 2. Top view of antimonene crystal structure with the hopping parameters $\left(t_{i}\right)$ included in the TB model. Their corresponding values are given in Table I. The red circles represent $p$ orbitals.

symmetry point of the BZ, with relevant contributions from all three $p$ orbitals of $\mathrm{Sb}$.

Since we are interested in electronic properties of semiinfinite ribbons, the momentum parallel to the infinite edge is a good quantum number and we can Fourier transform Hamiltonian (1) along that direction. The band structure of finite nanoribbons (we impose periodic boundary conditions along the direction parallel to the edge) with zigzag and armchair termination is shown in Fig. 3. First, the finite width of the antimonene ribbon leads to a reconstruction of the band structure with the formation of electronic bands composed of the accumulation of $N$ subbands, where $N$ is the number of unit cells along the width of the ribbon. Second, midgap edge states appear in both armchair and zigzag nanoribbons (Fig. 3), originating from the unsaturated bond on the edge of the ribbon. This is different from graphene and black phosphorus ribbons, for which edge states are absent for armchair termination [21,24]. The energy bands associated to the edge states are flat and weakly dispersing, leading to

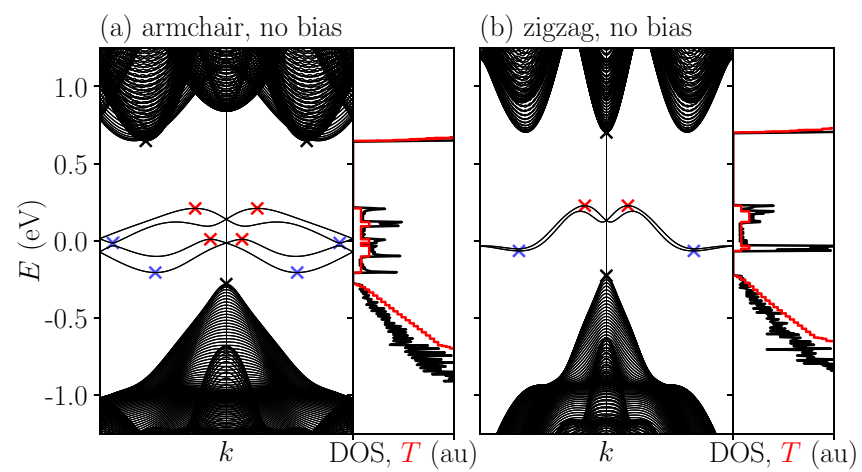

FIG. 3. Band structure, DOS, and transmission for (a) an armchair ribbon of width $41 \mathrm{~nm}$ and (b) a zigzag ribbon of width $36 \mathrm{~nm}$. The conduction- and valence-band edges are indicated with black crosses, the edge band maxima are red, and the edge band minima are blue. The midgap bands correspond to edge states. 

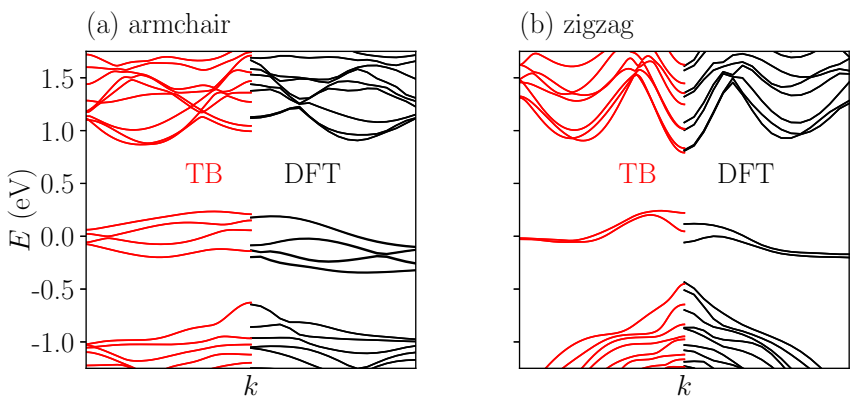

FIG. 4. Comparison of the band structure of nanoribbons as obtained from tight-binding and DFT methods: (a) a 2.3-nm-width ribbon with armchair termination and (b) a 2.9-nm-width ribbon with zigzag termination. Red corresponds to TB method and black to DFT calculations.

prominent peaks in the DOS associated to saddle points in the band structure.

In this work, we only consider chemically unsaturated edges, i.e., we do not take edge chemistry into account. Attaching different atoms to the edge could significantly alter the electronic structure around the Fermi level [25].

To check whether our bulk TB model also agrees with $a b$ initio calculations for finite ribbons, we performed first-principles calculations on the electronic structure of antimony nanoribbons, including SOC, using the Vienna $a b$ initio simulation package (VASP) [26,27]. Electron exchange and correlation interactions were described using the Perdew-Burke-Ernzerhof (PBE) pseudopotentials within the projector augmented-wave method [28]. The Brillouin-zone sampling was done using a $35 \times 1 \times 1$ Monkhorst-Pack grid for static calculation. The atomic structure of the nanoribbons is obtained from the $2 \mathrm{D}$ nanosheet without structure relaxation, and the vacuum region between two adjacent images is set to be $100 \AA$. The results are shown in Fig. 4 in comparison to tight-binding calculations. We can see that the agreement between the two methods is reasonable. Apart from some slight shifts in energies, the contours of the conduction-band minimum (CBM), valence-band maximum (VBM), and edge states in the tight-binding (TB) model are very similar to the density functional theory (DFT) result. We notice that previous first-principles calculations for narrow nanoribbons, of up to $\sim 3.4 \mathrm{~nm}$, predicted a direct band gap for zigzag termination [20], which is also in agreement with our own TB calculations. By systematically studying the evolution of the band gap with nanoribbon size, we conclude that the two secondary CBMs around $k= \pm 0.63 \frac{\pi}{W}$ get closer to the CBM at $k=0$ for increasing size. For a ribbon width of $175 \mathrm{~nm}$, the difference between their energy values is only of the order of $10^{-5} \mathrm{eV}$.

The midgap states are highly localized at the edges, as can be seen in Fig. 5. For zero bias, each $\mathbf{k}$ presents two degenerate states with opposite spin in opposite sides of the ribbon. Since time-reversal symmetry must be preserved, the spin polarization of one edge associated to one state of a given wave vector $\mathbf{k}$ is compensated by the opposite spin polarization of the degenerate state with momentum $-\mathbf{k}$.

In the following, we use Landauer theory [29] to calculate the electronic transmission in the scattering-free limit, which (a) armchair edge state

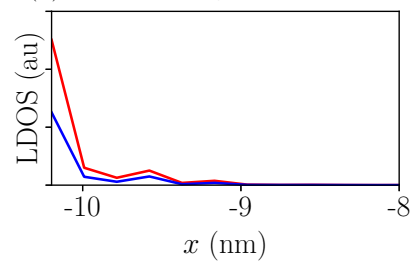

(b) zigzag edge state

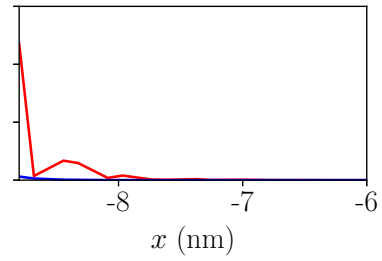

FIG. 5. LDOS of edge states at $k=\frac{\pi}{2 W}$ for (a) an armchair ribbon of width $20 \mathrm{~nm}$ and (b) a zigzag ribbon of width $18 \mathrm{~nm}$. The red and blue lines correspond to spin up and down, respectively. Notice that these states are degenerate with the corresponding states for $k=-\frac{\pi}{2 W}$, which have opposite spin.

is obtained by counting modes,

$$
T(E)=\sum_{k} N_{k}(E)
$$

where $N_{k}(E)$ is the number of bands that cross the energy $E$ for a given wave vector $k$. The results for $T(E)$ for each termination are shown in Fig. 3 (solid red lines), together with the DOS (solid black lines).

The main difference between the transmission in zigzag and armchair nanoribbons occurs for energies within the bulk band gap. At these energies, $T(E)$ is dominated by edge states which, as we have seen, are different for zigzag and armchair terminations. As the ribbon width increases, the transmission function corresponding to the bulk states increases, accompanied by a reduction of the energy gap, while the transmission of the edge states remains the same.

We have further calculated the effective mass of electrons $m_{e}^{*}$ and holes $m_{h}^{*}$ from the nanoribbon band structure (Table II). Electrons are heavier than holes for both edge terminations. We also find that carriers in zigzag nanoribbons are expected to have lower effective masses than in armchair nanoribbons. These results can be useful for calculations based on low-energy $\mathbf{k} \cdot \mathbf{p}$ analytical models.

\section{OUT-OF-PLANE BIAS}

The application of external gate voltages is a powerful tool to control and tune the electronic and optical properties of layered 2D materials [30]. In this section, we study the effect of a perpendicular bias voltage on antimonene. Since monolayer $\mathrm{Sb}$ is buckled, the application of an electric field perpendicular to the sample leads to a potential difference between atoms in different planes. Therefore, we introduce an out-of-plane bias $\Delta V_{P}$ (without considering screening) by setting the on-site potential on the two sublattices in Hamiltonian (1) to different values,

$$
\epsilon_{\text {mi }}=\Delta V_{P} \times z_{i}
$$

TABLE II. Effective masses for antimonene nanoribbons.

\begin{tabular}{lcc}
\hline \hline Edge & $m_{e}^{*}\left(m_{0}\right)$ & $m_{h}^{*}\left(m_{0}\right)$ \\
\hline Armchair & 0.2 & 0.13 \\
Zigzag & 0.13 & 0.09 \\
\hline \hline
\end{tabular}



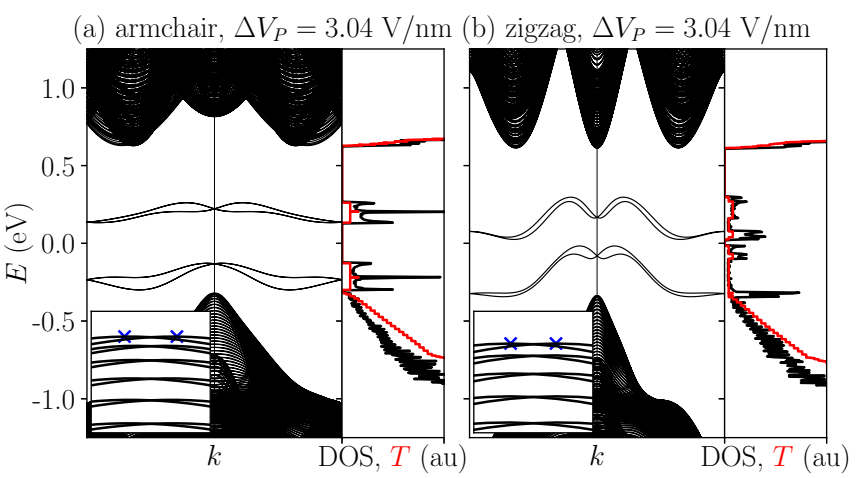

FIG. 6. Top: band structure, DOS and transmission with $\Delta V_{P}=$ $3.04 \mathrm{~V} / \mathrm{nm}$ for (a) an armchair ribbon of width $41 \mathrm{~nm}$ and (b) a zigzag ribbon of width $36 \mathrm{~nm}$. Insets: close up of the edge of the valence band, with blue crosses to indicate the maxima.

where $z_{i}$ is the $z$ coordinate of site $i$ in the buckled structure, which is plus or minus $0.82 \AA$ on sublattice $A$ or $B$, respectively. Our results for the zigzag and armchair nanoribbon band structure, with the corresponding DOS and electronic transmission, are given in Fig. 6. First, we notice that for both types of ribbon, we obtain a band-gap widening under the application of the electric field. The evolution with the applied bias of the valence and conduction bands, as well as the edge states, are shown in Fig. 7. Opening of the band gap with electric field was also predicted for single-layer black phosphorus [31]. Interestingly, application of a bias voltage breaks inversion symmetry (sublattices $A$ and $B$ are no longer equivalent). This, together with the strong spin-orbit coupling leads, due to Rashba effect, to splitting of the edge states and of the valence and conduction bands. Notice that because of the latter, the zigzag ribbon band gap becomes indirect when a bias is applied (see insets of Fig. 6 for a closeup of the valence-band edge). The application of a perpendicular bias field, therefore, opens the possibility to dynamically tune the Rashba energy [32] or to study unconventional transport prop-
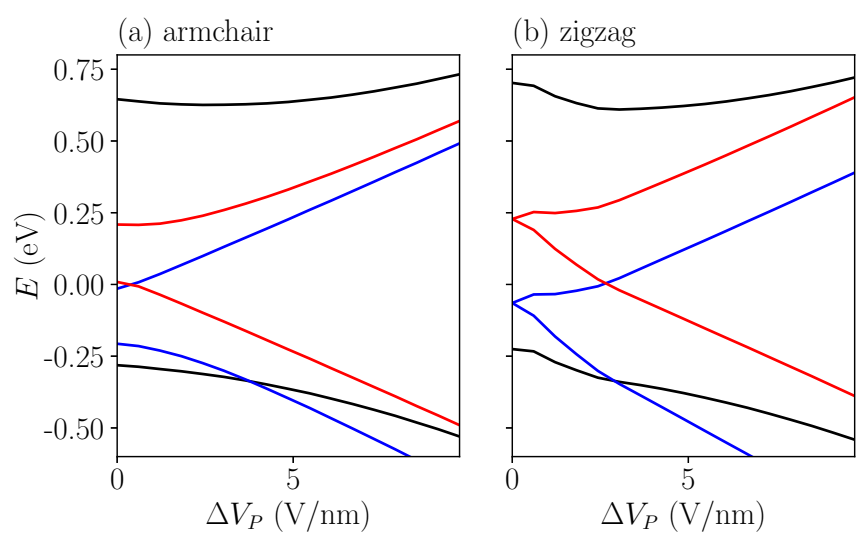

FIG. 7. Band edges as a function of $\Delta V_{P}$ for (a) an armchair ribbon of width $41 \mathrm{~nm}$ and (b) a zigzag ribbon of width $36 \mathrm{~nm}$. Black lines correspond to the conduction- and valence-band edges, and red (blue) corresponds to the maxima (minima) of the edge states, corresponding to the crosses in Fig. 3.

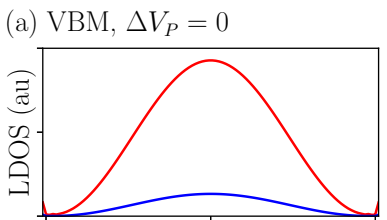

(c) left VBM, $\Delta V_{P}=1.82 \mathrm{~V} / \mathrm{nm}$

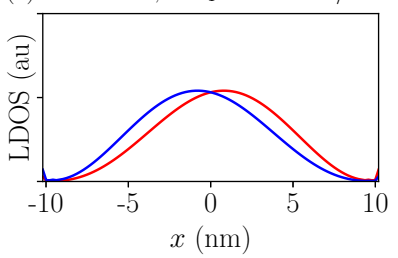

(b) VBM, $\Delta V_{P}=0$

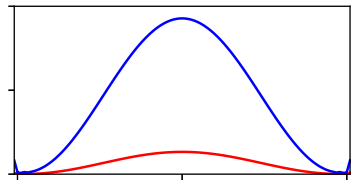

(d) right VBM, $\Delta V_{P}=1.82 \mathrm{~V} / \mathrm{nm}$

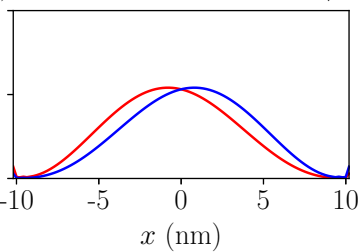

FIG. 8. LDOS of the valence-band maxima for an armchair ribbon of width $20 \mathrm{~nm}$, under out-of-plane bias. The red and blue lines correspond to spin up and down, respectively.

erties associated to entanglement between spin and charge degrees of freedom [33].

The local distribution of the eigenstates can be investigated by calculating the local density of states (LDOS) [34], which is the probability amplitude as a function of location, in the transverse direction of the ribbon. Our results for the LDOS corresponding to the valence band are shown in Fig. 8. First, we notice that in the absence of a bias field, the maximum of the valence band is doubly degenerate (due to spin). The local distribution of the VB states is maximum at the center of the ribbon and decays as we approach the ribbon edges [Figs. 8(a) and $8(\mathrm{~b})$ ]. The situation is different when an out-of-plane electric field is applied: as discussed above, due to Rashba coupling the edge of the VB is split into two maxima around $\Gamma$ (see insets of Fig. 6) and the spin degeneracy is broken. The consequence of this on the LDOS is seen in Figs. 8(c) and $8(\mathrm{~d})$. The states corresponding to the left maximum [Fig. 8(c)] present a major contribution of spin down (up) at the left (right) side of the center of the ribbon. Of course, since time-reversal symmetry must be conserved, the opposite happens for the states associated to the right VBM [Fig. 8(d)].

For the armchair ribbon, for nonzero out-of-plane bias, the two lower midgap bands move down and the two upper bands move up. For the zigzag case, however, the two midgap bands, which were originally doubly degenerate, split into two pairs of nondegenerate bands. The edge states on one side of the ribbon move up in energy, while the states on the other side move down. This is due to the fact that the sites on one edge of the ribbon have a $z$ coordinate of $+0.82 \AA$ and, on the other edge, $-0.82 \AA$ because of the buckled structure.

\section{IN-PLANE TRANSVERSE BIAS}

Another possibility to tune the band structure is to apply an in-plane electric field. This way we create a transverse bias potential along the ribbon. To account for a transverse bias $\Delta V_{T}$, we set in Hamiltonian (1) the on-site energy

$$
\epsilon_{m i \sigma}=\Delta V_{T} \times x_{i},
$$

where $x_{i}$ is the coordinate of site $i$ in the transverse direction. Our results for two different values of bias field are given 

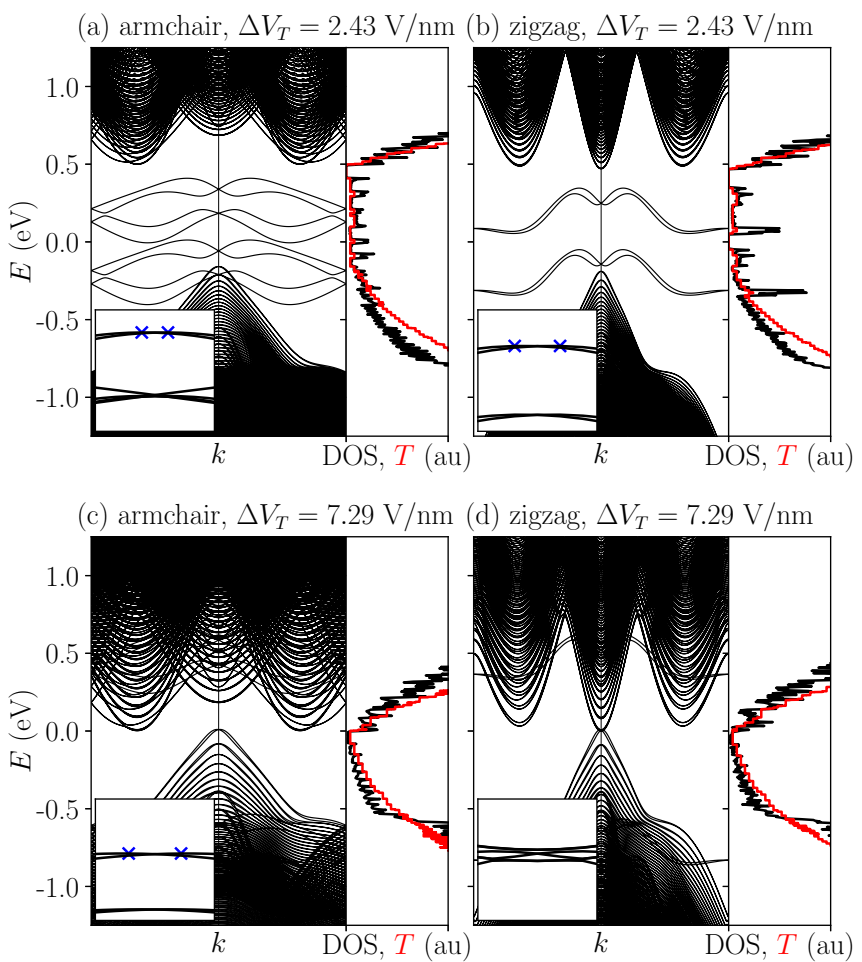

FIG. 9. Top: Band structure, DOS, and transmission for (a) an armchair ribbon of width $41 \mathrm{~nm}$ with $\Delta V_{P}=2.43 \mathrm{~V} / \mathrm{nm}$, (b) a zigzag ribbon of width $36 \mathrm{~nm}$ with $\Delta V_{P}=2.43 \mathrm{~V} / \mathrm{nm}$, (c) an armchair ribbon of width $41 \mathrm{~nm}$ with $\Delta V_{P}=7.29 \mathrm{~V} / \mathrm{nm}$, and (d) a zigzag ribbon of width $36 \mathrm{~nm}$ with $\Delta V_{P}=7.29 \mathrm{~V} / \mathrm{nm}$. Insets: Close up of the edge of the valence band, with blue crosses to indicate the maxima.

in Fig. 9. First, we observe a band-gap reduction for both types of edge termination. The evolution of the valence- and conduction-band edges, as well as the position of the extrema of the edge states, are shown in Fig. 10. Notice that the color
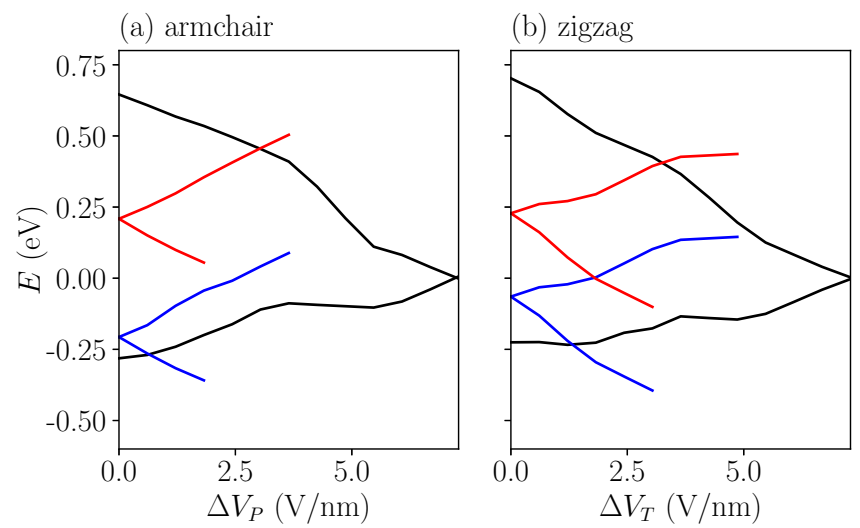

FIG. 10. Band edges as a function of $V_{T}$ for (a) an armchair ribbon of width $41 \mathrm{~nm}$ and (b) a zigzag ribbon of width $36 \mathrm{~nm}$. Black lines correspond to the conduction- and valence-band edges, and red (blue) corresponds to the maxima (minima) of the edge states. The curves corresponding to edge states are cut when such states can no longer be distinguished from the conduction- or valence-band states in the band structure.

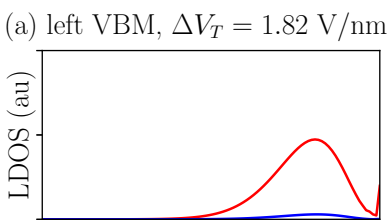

(c) left CBM, $\Delta V_{T}=1.82 \mathrm{~V} / \mathrm{nm}$

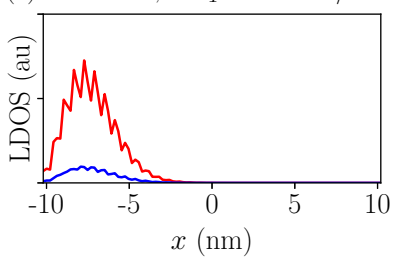

(b) right VBM, $\Delta V_{T}=1.82 \mathrm{~V} / \mathrm{nm}$

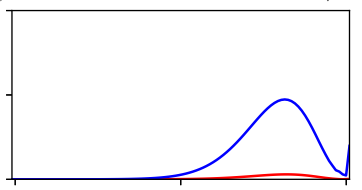

(d) right CBM, $\Delta V_{T}=1.82 \mathrm{~V} / \mathrm{nm}$

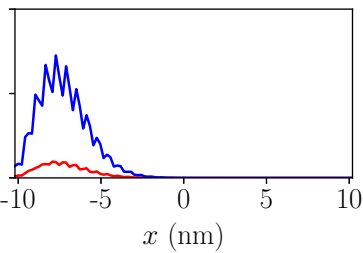

FIG. 11. LDOS of the valence-band maxima for an armchair ribbon of width $20 \mathrm{~nm}$, under transverse bias. The red and blue lines correspond to spin up and down, respectively.

lines, corresponding to the edge states, are cut when such states can no longer be distinguished from the conduction- or valence-band states in the band structure. Similarly as in the case of an out-of-plane field, inversion symmetry is broken along the ribbon and the edge states are split (see insets of Fig. 9).

The main difference with respect to the case of out-ofplane bias is that the edges of the valence and conduction bands correspond to states that are located at the edges of the ribbon. This is clearly seen in the LDOS calculations (Fig. 11). While the states that form the valence-band edge are placed at the right edge [see Figs. 11(a) and 11(b)], the conduction-band edge is located at the left edge of the ribbon, as can be seen in Figs. 11(c) and 11(d).

As the sites on the edges gain an on-site potential $\pm \Delta V_{T} \frac{W}{2}$, the edge states on one side of the ribbon move up in energy, while on the other side they move down. Otherwise, the shape of the edge states stays the same.

These results are similar to those obtained for a black phosphorus nanoribbon in the presence of a transverse electric field [19]. The local separation between the conduction- and valence-band states can be quantified by calculating the polarization [35] $P=e \sum_{m i \sigma} \mathbf{r}_{i} c_{m i \sigma}^{\dagger} c_{m i \sigma}$, which yields $\langle P\rangle_{\mathrm{VBM}}=$ $5.61 e \mathrm{~nm}$ and $\langle P\rangle_{\mathrm{CBM}}=-7.25 e \mathrm{~nm}$ for this configuration.

Moreover, applying a transverse bias causes the valence band to split, lifting the degeneracy of the predominantly spin-up and spin-down states at the VBM. This is due to the fact that Rashba coupling is also present in this case (see insets in Fig. 9), which leads to different spin polarization of the two extrema of the valence band, represented by different color of the two VBM [Figs. 11(a) and 11(b)]. The armchair CBM also becomes spin polarized. For the zigzag case and for ribbons with $W<62 \mathrm{~nm}$, the CBM is at $k=0$, where there is no spin polarization.

\section{CONCLUSION}

In summary, we have studied the electronic properties of antimonene nanoribbons, in the presence of out-of-plane and in-plane electric fields, using a tight-binding model. We 
have shown that there is good agreement between $a b$ initio results and the tight-binding model. Our calculations show that antimonene nanoribbons are semiconducting in their bulk, i.e., not taking edge states into account. We have found that contrary to phosphorene, both kinds of termination, i.e., zigzag and armchair, present edge states inside the gap. Under the application of external bias fields, we have demonstrated that the gap can be enhanced by applying an out-of-plane bias. Under a transverse in-plane electric field, the gap decreases. Furthermore, a transverse bias leads to spatial separation between the states forming the edges of the valence and conduction bands. Both types of bias cause valence-band splitting due to Rashba coupling induced by lack of inversion symmetry. Such splitting is accompanied by a different spin polarization of the two minivalleys at both sides of the $\Gamma$ point.

\section{ACKNOWLEDGMENTS}

J.Y. acknowledges financial support from MOST Grant No. 2017YFA0303404, NSAF Grant No. U1530401, and computational resources from the Beijing Computational Science Research Center. M.I.K. acknowledges financial support from the European Research Council Advanced Grant program (Contract No. 338957). S.Y. acknowledges financial support from the Thousand Young Talent Plan (China). R.R. acknowledges financial support from the Spanish MINECO through the Ramón y Cajal program, Grants No. RYC-2016-20663 and No. FIS2014- 58445-JIN.
[1] K. S. Novoselov, D. Jiang, F. Schedin, T. J. Booth, V. V. Khotkevich, S. V. Morozov, and A. K. Geim, Two-dimensional atomic crystals, Proc. Natl. Acad. Sci. USA 102, 10451 (2005).

[2] R. Roldán, L. Chirolli, E. Prada, J. A. Silva-Guillén, P. SanJose, and F. Guinea, Theory of $2 \mathrm{~d}$ crystals: graphene and beyond, Chem. Soc. Rev. 46, 4387 (2017).

[3] J. Yu, M. I. Katsnelson, and S. Yuan, Tunable electronic and magneto-optical properties of monolayer arsenene from $\mathrm{GW}_{0}$ approximation to large-scale tight-binding simulations, Phys. Rev. B 98, 115117 (2018).

[4] S. Zhang, S. Guo, Z. Chen, Y. Wang, H. Gao, J. Gómez-Herrero, P. Ares, F. Zamora, Z. Zhu, and H. Zeng, Recent progress in 2D group-VA semiconductors: From theory to experiment, Chem. Soc. Rev. 47, 982 (2018).

[5] S. Zhang, Z. Yan, Y. Li, Z. Chen, and H. Zeng, Atomically thin arsenene and antimonene: semimetal-semiconductor and indirect-direct band-gap transitions, Angew. Chem., Int. Ed. 127, 3155 (2015).

[6] J. Ji, X. Song, J. Liu, Z. Yan, C. Huo, S. Zhang, M. Su, L. Liao, W. Wang, Z. Ni, Y. Hao, and H. Zeng, Two-dimensional antimonene single crystals grown by van der Waals epitaxy, Nat. Commun. 7, 13352 (2016).

[7] P. Ares, F. Aguilar-Galindo, D. Rodríguez-San-Miguel, D. A. Aldave, S. Díaz-Tendero, M. Alcamí, F. Martín, J. GómezHerrero, and F. Zamora, Mechanical isolation of highly stable antimonene under ambient conditions, Adv. Mater. 28, 6332 (2016).

[8] C. Gibaja, D. Rodriguez-San-Miguel, P. Ares, J. GómezHerrero, M. Varela, R. Gillen, J. Maultzsch, F. Hauke, A. Hirsch, G. Abellán, and F. Zamora, Few-layer antimonene by liquid-phase exfoliation, Angew. Chem., Int. Ed. 55, 14345 (2016).

[9] X. Wu, Y. Shao, H. Liu, Z. Feng, Y.-L. Wang, J.-T. Sun, C. Liu, J.-O. Wang, Z.-L. Liu, S.-Y. Zhu, Y.-Q. Wang, S.-X. Du, Y.-G. Shi, K. Ibrahim, and H.-J. Gao, Epitaxial growth and airstability of monolayer antimonene on $\mathrm{PdTe}_{2}$, Adv. Mater. 29, 1605407 (2017).

[10] L. Lu, X. Tang, R. Cao, L. Wu, Z. Li, G. Jing, B. Dong, S. Lu, Y. Li, Y. Xiang, J. Li, D. Fan, and H. Zhang, Broadband nonlinear optical response in few-layer antimonene and antimonene quantum dots: A promising optical Kerr media with enhanced stability, Adv. Opt. Mater. 5, 1700301 (2017).
[11] G. Wang, R. Pandey, and S. P. Karna, Atomically thin groupV elemental films: Theoretical investigations of antimonene allotropes, ACS Appl. Mater. Interfaces 7, 11490 (2015).

[12] O. Üzengi Aktürk, V. Ongun Özçelik, and S. Ciraci, Singlelayer crystalline phases of antimony: Antimonenes, Phys. Rev. B 91, 235446 (2015).

[13] D. Singh, S. K. Gupta, Y. Sonvane, and I. Lukačević, Antimonene: A monolayer material for ultraviolet optical nanodevices, J. Mater. Chem. C 4, 6386 (2016).

[14] G. Pizzi, M. Gibertini, E. Dib, N. Marzari, G. Iannaccone, and G. Fiori, Performance of arsenene and antimonene doublegate MOSFETs from first principles, Nat. Commun. 7, 12585 (2016).

[15] Y. Xu, B. Peng, H. Zhang, H. Shao, R. Zhang, and H. Zhu, First-principle calculations of optical properties of monolayer arsenene and antimonene allotropes, Ann. Phys. (Berlin) 529, 1600152 (2017).

[16] A. N. Rudenko, M. I. Katsnelson, and R. Roldán, Electronic properties of single-layer antimony: Tight-binding model, spinorbit coupling, and the strength of effective Coulomb interactions, Phys. Rev. B 95, 081407 (2017).

[17] Y. Wang, P. Huang, M. Ye, R. Quhe, Y. Pan, H. Zhang, H. Zhong, J. Shi, and J. Lu, Many-body effect, carrier mobility, and device performance of hexagonal arsenene and antimonene, Chem. Mater. 29, 2191 (2017).

[18] H. Guo, N. Lu, J. Dai, X. Wu, and X. C. Zeng, Phosphorene nanoribbons, phosphorus nanotubes, and van der waals multilayers, J. Phys. Chem. C 118, 14051 (2014).

[19] E. Taghizadeh Sisakht, M. H. Zare, and F. Fazileh, Scaling laws of band gaps of phosphorene nanoribbons: A tight-binding calculation, Phys. Rev. B 91, 085409 (2015).

[20] Y. Wang and Y. Ding, Electronic structure and carrier mobilities of arsenene and antimonene nanoribbons: A first-principle study, Nano. Res. Lett. 10, 254 (2015).

[21] M. M. Grujić, M. Ezawa, M. Z. Tadić, and F. M. Peeters, Tunable skewed edges in puckered structures, Phys. Rev. B 93, 245413 (2016).

[22] Y. Song, X. Wang, and W. Mi, Spin splitting and electric field modulated electron-hole pockets in antimonene nanoribbons, npj Quantum Mater. 2, 15 (2017).

[23] H.-S. Tsai, C.-W. Chen, C.-H. Hsiao, H. Ouyang, and J.-H. Liang, The advent of multilayer antimonene nanoribbons with 
room temperature orange light emission, Chem. Commun. 52, 8409 (2016).

[24] L. Brey and H. A. Fertig, Electronic states of graphene nanoribbons studied with the Dirac equation, Phys. Rev. B 73, 235411 (2006).

[25] D. Gunlycke, J. Li, J. W. Mintmire, and C. T. White, Altering low-bias transport in zigzag-edge graphene nanostrips with edge chemistry, Appl. Phys. Lett. 91, 112108 (2007).

[26] G. Kresse and J. Furthmüller, Efficient iterative schemes for ab initio total-energy calculations using a plane-wave basis set, Phys. Rev. B 54, 11169 (1996).

[27] G. Kresse and J. Furthmüller, Efficiency of ab initio total energy calculations for metals and semiconductors using a plane-wave basis set, Comput. Mater. Sci. 6, 15 (1996).

[28] P. E. Blöchl, Projector augmented-wave method, Phys. Rev. B 50, 17953 (1994).

[29] R. Landauer, Spatial variation of currents and fields due to localized scatterers in metallic conduction, IBM J. Res. Dev. 1, 223 (1957).
[30] R. Roldán and A. Castellanos-Gomez, Black phosphorus: A new band-gap tuning knob, Nat. Photon. 11, 407 (2017).

[31] S. Yuan, E. van Veen, M. I. Katsnelson, and R. Roldán, Quantum Hall effect and semiconductor-to-semimetal transition in biased black phosphorus, Phys. Rev. B 93, 245433 (2016).

[32] C. R. Ast, D. Pacilé, L. Moreschini, M. C. Falub, M. Papagno, K. Kern, M. Grioni, J. Henk, A. Ernst, S. Ostanin, and P. Bruno, Spin-orbit split two-dimensional electron gas with tunable Rashba and Fermi energy, Phys. Rev. B 77, 081407 (2008).

[33] V. Brosco, L. Benfatto, E. Cappelluti, and C. Grimaldi, Unconventional dc Transport in Rashba Electron Gases, Phys. Rev. Lett. 116, 166602 (2016).

[34] H. Rostami, R. Asgari, and F. Guinea, Edge modes in zigzag and armchair ribbons of monolayer $\mathrm{MoS}_{2}$, J. Phys.: Condens. Matter 28, 495001 (2016).

[35] S. Yuan, H. De Raedt, and M. I. Katsnelson, Modeling electronic structure and transport properties of graphene with resonant scattering centers, Phys. Rev. B 82, 115448 (2010). 